\title{
Grating Coupler with Integrated Power Splitter for High-Intensity Optical Power Distribution
}

\author{
T. Spuesens, S. Pathak, M. Vanslembrouck, P. Dumon and W. Bogaerts
}

\begin{abstract}
In this paper we present a novel fiber grating coupler with integrated 16-way power splitter. The incoming light from the fiber is split immediately over 16 channels and therefore the total optical power is never confined in a single waveguide. This is of particular interest for silicon photonics platforms because here high optical intensities can cause significant nonlinear losses. The device has a total coupling efficiency that is similar to standard focusing grating couplers. Furthermore, a channel non-uniformity below $1.1 \mathrm{~dB}$ has been obtained. By studying the alignment sensitivity, we found that for high splitting uniformity a careful positioning of the fiber is necessary. We also experimentally demonstrate that this device is indeed capable of handling high optical powers without introducing additional non-linear losses.
\end{abstract}

\section{INTRODUCTION}

Realizing efficient on-chip optical sources on a silicon photonics platform remains one of the biggest hurdles of this technology. As an alternative, an off-chip optical light source can be used as an optical power supply for the rest of the chip. However, the cost of external laser integration is significant and therefore it would be desirable to reduce the number of such lasers and use an on-chip splitter network to feed multiple channels with each laser [1]. Obviously, such an approach requires high optical power at the input of the distribution network. It is well known however, that in silicon the optical power that can be efficiently guided in a submicron wire waveguide is limited by two-photon absorption and subsequently free carrier absorption. It can be calculated, and we experimentally verified, that these effects already kick in around $10 \mathrm{~mW}$ in $450 \mathrm{~nm}$ wide silicon strip waveguides, and at $50 \mathrm{~mW}$ one has to account for $3 \mathrm{~dB} / \mathrm{cm}$ additional loss due to these non-linear processes. This effect can of course be mitigated to some extent by using wider waveguides or by using rib waveguides to lower the maximum intensity, but even then this will not significantly increase the power limits for the input waveguide of the distribution network that needs to deal with the total optical power.

Here we propose a novel coupling scheme where the coupler immediately distributes the external optical power over $N$ waveguides ( $N=16$ in our case). The total optical power is therefore never fully confined in a submicron waveguide core,

T. Spuesens, M. Vanslembrouck and W. Bogaerts are with the Photonics Research Group, Department of Information Technology, imec and Center for Nano- and Biophotonics, Ghent University, Ghent, Belgium. W. Bogaerts is also with Luceda Photonics, Dendermonde, Belgium. (email: thijs.spuesens@intec.ugent.be)

S. Pathak is with University of California Davis, Dept. of Electrical and Computer Engineering, Davis, CA 95616, USA

P. Dumon is with Luceda Photonics, Dendermonde, Belgium. avoiding high intensities. To achieve this, we have engineered a variation on the fiber grating coupler, which is a frequently used component in silicon photonics [2]. The principle is shown in Figure 1: a curved grating diffracts the external light into the silicon slab, but unlike a traditional curved grating coupler, the light is not focused into a single waveguide [3], but rather spread out towards a set of apertures, similar to star couplers used in arrayed waveguide gratings (AWG) [4].

We already presented preliminary results of this concept at Group IV Photonics [5]. Here, we demonstrate the device in a full silicon photonics platform and give a more in-depth analysis of the sensitivity to fiber alignment and the device performance in high-power settings, demonstrating its unique advantage as a coupler for an 'optical power supply'.

\section{DESIGN}

The design consists of two parts: the grating and the star coupler. The grating is constructed from periodic confocal elliptical lines and is therefore similar to a focusing grating coupler [3]. However, in this case the fiber is not oriented towards the focal point, but rather away from it resulting in a diverging field in the silicon slab. The period of the grating is chosen such that maximum coupling efficiency is obtained for a wavelength of $1550 \mathrm{~nm}$ under a fiber angle of 10 degrees. Two different grating structures were used: a shallow etched grating in a $220 \mathrm{~nm}$ thick silicon layer, with a typical coupling efficiency of $-5 \mathrm{~dB}[3]$, and a high efficiency grating using a poly-silicon overlay, which yields $-2.5 \mathrm{~dB}$ per coupler [6].

The grating coupler operates for a single polarization (TE). For applications as an optical power supply this should not

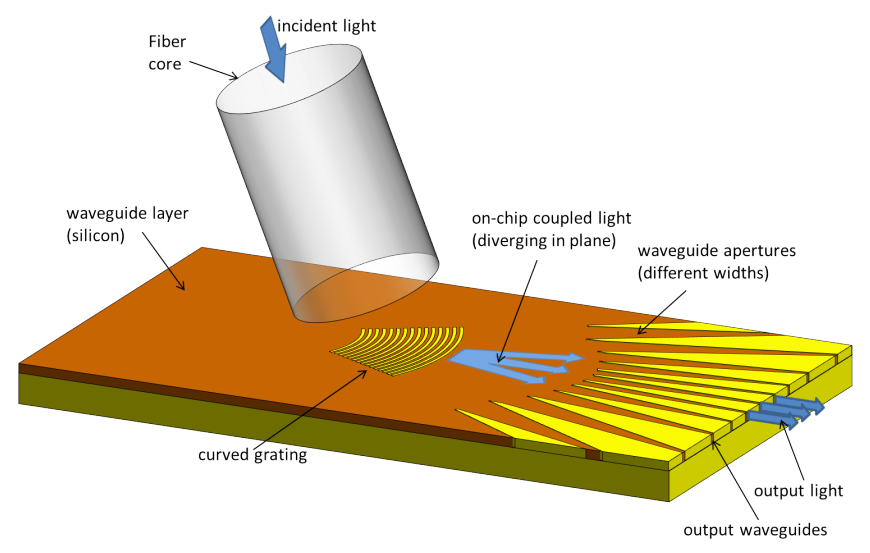

Fig. 1. Grating Coupler + Power splitter. The grating distributes the external light over the $\mathrm{N}$ output waveguides. 


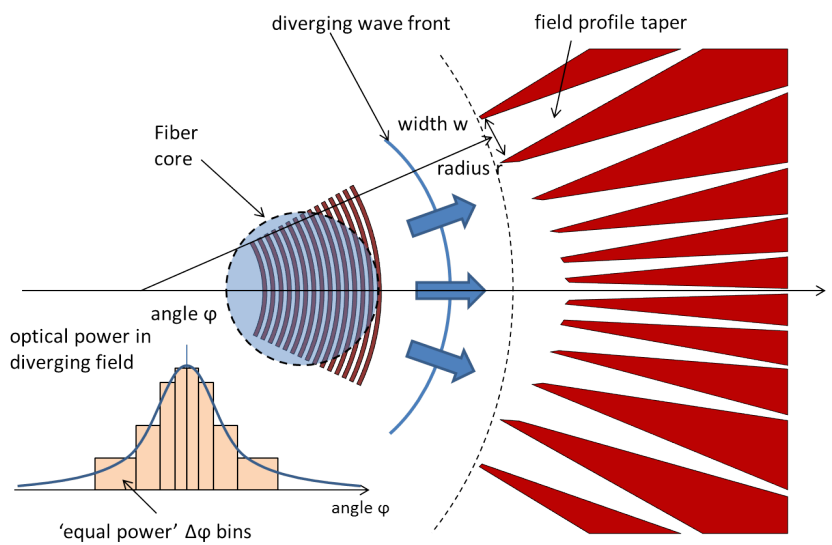

Fig. 2. Design of the star coupler. The angular spread of the diverging light is distributed over equal power bins.

present a drawback, as most lasers and silicon waveguide circuits are typically single-polarization.

To design the star coupler we start from a virtual input aperture that coincides with the focal point of the grating. This is the point from where the phase fronts diverge. We assume a Gaussian distribution of the optical power, corresponding to a fiber mode. A uniform array of output apertures will therefore result in a Gaussian distribution of power over these apertures. In most cases however, a homogeneous power distribution is desired in a power splitter. This can be achieved by modifying the individual output apertures. For this, we considered the radially diverging phase front and split it up into equal power bins along the in-plane angle $\phi$. One can now modify the radial position and width of the individual apertures to match the angular coverages of these power bins. This is illustrated in Figure 2. When a uniform width is used this will result in very short radii for the outer apertures and then the trenches can cast a shadow effect on the neighboring apertures. A uniform radius on the other hand, requires very wide outer apertures which will have an additional penalty because of poor modal overlap and the need for very long tapers. We can use a more balanced approach in which we modify both the width and radius of the apertures. For example, we mounted the apertures on a Rowland circle with adjusted widths, and we were able to obtain a highly uniform power distribution. The design and simulation of the device was done using the IPKISS parametric design framework from Luceda Photonics, and a Fresnel diffraction engine for the star coupler [4].

\section{FABRICATION AND CHARACTERIZATION}

\section{A. Fabrication}

The devices were fabricated in imec's silicon photonics full platform (ISIPP25G) on an SOI wafer with $220 \mathrm{~nm}$ silicon on a $2 \mu \mathrm{m}$ buried oxide. First, the passive waveguide layer is processed using three etch steps: A complete $220 \mathrm{~nm}$ etch, a partial 70nm etch and a partial 160nm etch. We used the deep etch for our output waveguides and the $70 \mathrm{~nm}$ etch to define the regular grating couplers and the trenches for the star coupler. These structures are oxide-clad and planarized, and on top an additional $160 \mathrm{~nm}$ of poly-silicon is deposited. This layer is then patterned to define the high-efficiency gratings. The final structure also includes a BEOL stack that contains e.g. metal layers for contacting active devices. The fabricated devices are shown in Figure 3. We fabricated the star coupler power splitters both for different trade-offs between aperture width and radius. As a reference, we also included a design with a uniform star coupler (all radii and aperture widths identical) which is expected to have a Gaussian-like power distribution.

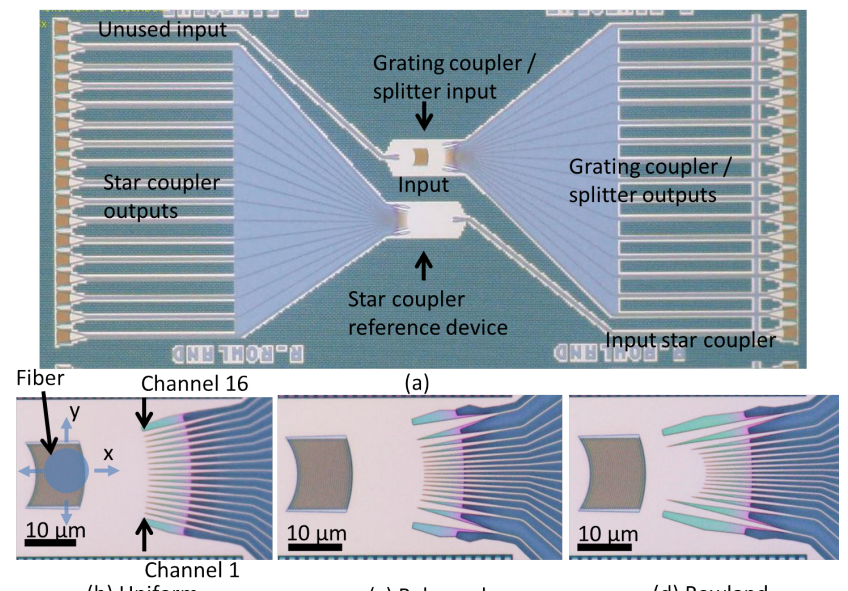

(b) Uniform

(c) Balanced

(d) Rowland

Fig. 3. (a) Microscope image of the two structures used to analyze the high power handling. The top device is an integrated grating coupler/splitter, while the lower device is a normal star coupler where the input aperture is connected with a single mode wire waveguide. Zoomed in microscope image of a uniform (b), balanced (c) and Rowland (d) star coupler design.

\section{B. Characterization method}

We characterized the devices using an automated twin-fiber probe station. Both fibers can be automatically positioned based on the design data. For the grating couplers in the center of the splitter, the position of the input fiber is optimized with the output fiber located on one of the center outputs. The input fiber is then kept in place while measuring the transmission on all the outputs. The result is normalized against the efficiency of a single-port grating coupler, which was either $-6.2 \mathrm{~dB}$ for a standard grating coupler or $-5.71 \mathrm{~dB}$ for a high efficiency grating coupler, where the high value of the latter seems to be caused by a design error.

\section{EXPERIMENTAL RESULTS}

Figure 4 shows the normalized transmission for each of the 16 channels of the power splitter. It is clear that for a uniform star coupler we indeed get a Gaussian distribution over the output channels. With a "balanced" or a "Rowland" configuration however, a more uniform power distribution is obtained in both cases.

The "balanced" configuration yields a maximum power imbalance of only $1.11 \mathrm{~dB}$. Furthermore, it is found that the total coupling efficiency is $2.48 \mathrm{~dB}$ higher than that of a raised focusing grating coupler, at $-3.23 \mathrm{~dB}$. This is possible because the power is collected over a larger area in case of the defocusing grating coupler, making it more tolerant to 
imperfections in e.g. the grating teeth. A slightly higher total efficiency, but with a somewhat larger imbalance, was found for the "Rowland" configuration.

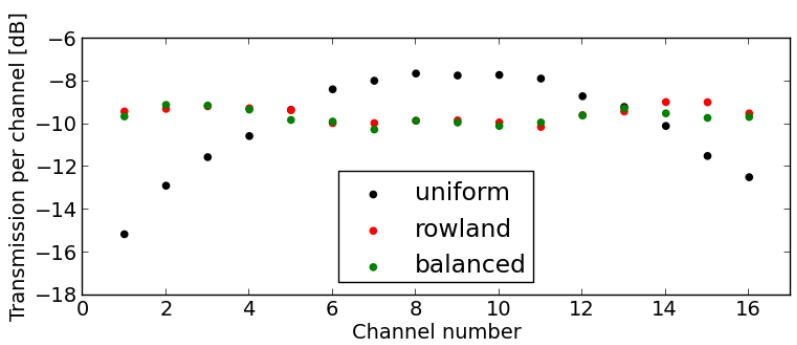

Fig. 4. Output power in each channel for different designs of defocusing grating coupler enabled power splitters.

\section{A. Alignment sensitivity}

We also assessed the sensitivity to fiber misalignment. The output channels are numbered as indicated in Figure 3(a). As a reference, we use the optimal fiber alignment for output 9 , as this is one of the most centered outputs. The position of the output fiber is now kept fixed on one of the outputs, while we scan the XY position of the input fiber with steps of 500 $\mathrm{nm}$ over a $10 \times 10 \mu \mathrm{m}$ area around the reference position. This results in a 2D map of power transmission as function of input fiber position. We repeat this process for all output channels and the results are plotted in Fig. 5(a-d) for channel 1, 8, 9 and 16. These center and outer channels represent the extreme cases. As can be seen, when alignment is performed with respect to one of the two most centered channels ( 8 and 9 ), the optimum position is centered around this reference position. If, on the other hand, one of the outermost channels is used for alignment, maximum power transfer is obtained at a strong offset in the vertical direction.

Figure 5(e) shows the impact of alignment on channel power uniformity when the input fiber is aligned with respect to a specific output channel. The lowest uniformity is obtained when the input fiber alignment is performed on one of the outermost channels and is almost as high as $20 \mathrm{~dB}$. If on the other hand, one of the two center channels are used, the nonuniformity is only $1.1 \mathrm{~dB}$.

In principle, the best uniformity can be obtained by simultaneously monitoring the output power on the two outermost channels. When the output power on these channels is equal the non-uniformity reaches a minimum that is determined by the widths and radial positions of the apertures and is in fact the real non-uniformity of the device. However, as can be seen from Figure 5(e), good balance can also be obtained by aligning on one of the two center channels.

To further quantify the alignment sensitivity in the vertical direction, we start by plotting again the power transmission for every channel when the input fiber is set to its zero position. Next to this, we also plot the results for deliberate offsets in the y direction with steps of $500 \mathrm{~nm}$. The result is shown in Figure 6. The impact for a $500 \mathrm{~nm}$ y-offset is limited for the center channels while it results in a power penalty of about 1 $\mathrm{dB}$ for the outer channels.
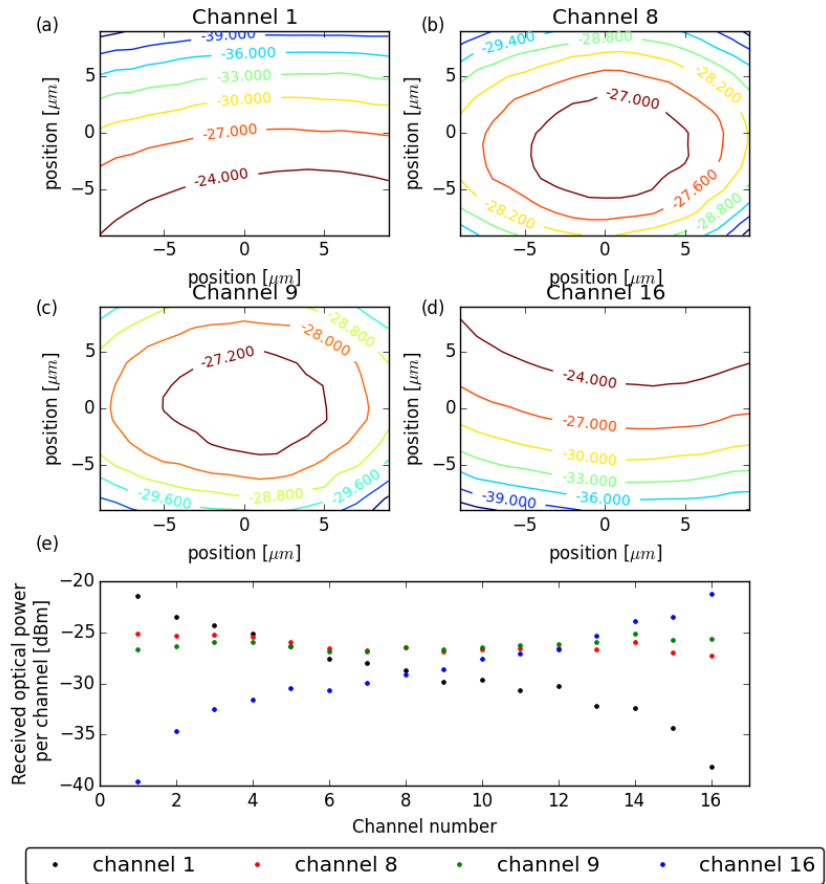

Fig. 5. Experimental result of alignment sensitivity measurements with a 2D contour map for channel 1, 8, 9 and 16 in (a-d), respectively. (e) Effect on splitting uniformity when alignment procedure is performed on output channel $1,8,9$ or 16

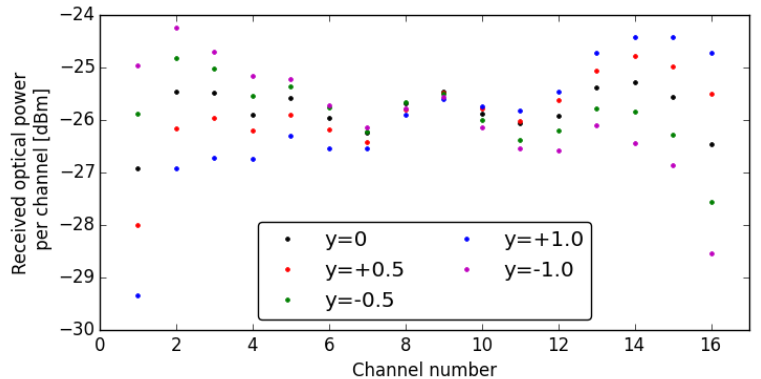

Fig. 6. Effect of fiber misalignment in the y-direction on the splitting uniformity.

\section{B. High power handling}

An important advantage of our device is its capability of coupling high optical powers. In order to verify this, we compared our device with a circuit consisting of a single focusing grating coupler connected to an on-chip star coupler with a $450 \mu \mathrm{m}$ long single-mode waveguide. A microscope image of these two test structures is shown in Figure 3. For both our test structures we perform a transmission measurement at low and high optical input power. For our proposed device we expect no significant additional loss, while for our reference structure with the single mode input waveguide it is expected that non-linear processes will introduce a significant additional loss.

For the optical input we used a Santec TL-510 fixed at a wavelength of $1570 \mathrm{~nm}$. In order to generate sufficiently high 
optical power the TL was followed by an OptoLink booster EDFA and an optical filter with a bandwidth of $3 \mathrm{~nm}$. A 99/1 splitter was inserted between the optical filter and the chip to monitor the optical power injected into the chip. The power transmission is then measured at all output channels with an HP power meter. The high power level was fixed to $275 \mathrm{~mW}$ $(24.4 \mathrm{dBm})$, while for the low power level we used $4 \mathrm{~mW}$ $(6 \mathrm{dBm})$. If there are no additional losses due to non-linear processes we therefore should see a difference of $18.4 \mathrm{~dB}$ in transmitted power between the measurement at low and high optical powers.

The measurement was performed on test structures with normal curved grating couplers. Therefore, the optical power coupled into the slab or waveguide is estimated at $66 \mathrm{~mW}$ for the high-power scenario. This is sufficiently high to observe losses caused by non-linear processes. A reference measurement on a $450 \mathrm{~nm}$ wide single mode waveguide of $1 \mathrm{~cm}$ length, indicated that the additional loss due to non-linear processes is about $3 \mathrm{~dB} / \mathrm{cm}$ for $50 \mathrm{~mW}$ of power in the waveguide. Obviously, because the loss depends on the optical power in the waveguide it is not evenly distributed over the full length of the waveguide, but is high at the start of the waveguide and then decays as the losses accumulate. Therefore, although the single mode input waveguide of the reference structure is only $450 \mu \mathrm{m}$ long, we still expect an additional loss close to $3 \mathrm{~dB}$. Figure 7(a) shows the measured transmission versus channel number for low and high optical input powers in the reference structure. It can be seen that the average transmitted power for the low and high optical input power only differs by $14.93 \mathrm{~dB}$ instead of $18.4 \mathrm{~dB}$, which means that the additional loss due to non-linear processes is $3.6 \mathrm{~dB}$ in this case. The same plot, but now for our new proposed device, is shown in Figure 7(b). In this case, the difference between the average transmitted power for low and high optical input power is $17.95 \mathrm{~dB}$, which implies an additional loss of $0.45 \mathrm{~dB}$. This is substantially lower compared to our reference case and shows that our proposed device is indeed able to handle high optical powers. It can also be seen in Figure 7(b) that at high optical input powers, the channel uniformity is slightly different from the measurement at low optical input powers. This is most likely caused by a small drift of the fiber due to thermal effects at these high powers. It is very well possible this drift also contributes to the measured additional loss of $0.45 \mathrm{~dB}$.

\section{CONClusion}

We have demonstrated an integrated grating coupler and 16-way power splitter. A power uniformity over 16 channels better than $1.11 \mathrm{~dB}$ has been achieved and the device has an efficiency similar to standard focusing grating couplers. While the influence of fiber alignment on the total efficiency is comparable with that of normal focusing grating couplers, the influence on splitting uniformity is strong and thus a careful alignment procedure is required. However, the 1.1 $\mathrm{dB}$ non-uniformity reported here was achieved by a simple alignment procedure on one of the center output channels. It was experimentally verified that the device is capable of handling high optical powers. Up to $275 \mathrm{~mW}$ of optical power
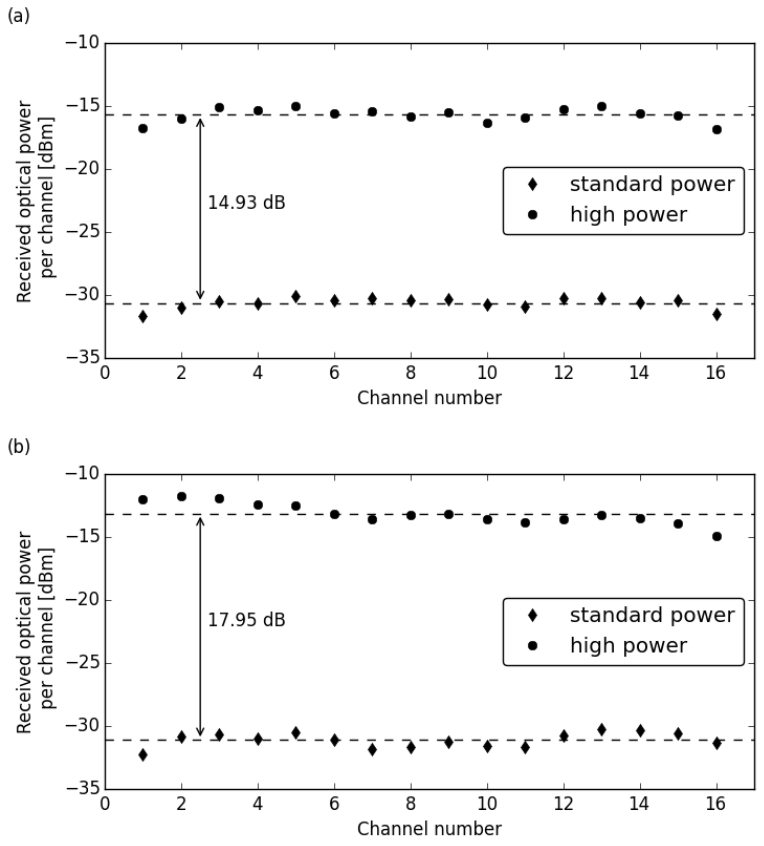

Fig. 7. Output power in each channel for low and high input power levels using (a) a standard star coupler and (b) the novel integrated grating coupler/splitter.

was injected on the grating coupler, resulting in $66 \mathrm{~mW}$ coupled into the silicon plane. This amount of power typically results in more than $3 \mathrm{~dB}$ additional loss in a $450 \mathrm{~nm}$ wide single mode waveguide but we did not observe any significant additional loss.

\section{ACKNOWLEDGMENT}

Part of this work was supported by the European Union through the Plat $4 \mathrm{M}$ project.

\section{REFERENCES}

[1] L. Vivien, S. Lardenois, D. Pascal, S. Laval, E. Cassan, J. L. Cercus, A. Koster, J. M. Fedeli and M. Heitzmann, "Experimental demonstration of a low-loss optical $\mathrm{H}$-tree distributionusing silicon-on-insulator microwaveguides" Appl. Phys. Lett., vol. 85, no. 5, pp. 701-703, Aug 2004.

[2] D. Taillaert, F. Van Laere, M. Ayre, W. Bogaerts, D. VanThourhout, P. Bienstman and R. Baets, "Grating Couplers for Coupling between Optical Fibers and Nanophotonic Waveguides" Jpn. J. Appl. Phys., vol. 45, no. 8A, pp. 6071-6077, 2006.

[3] F. Van Laere, T. Claes, J. Schrauwen, S. Scheerlinck, W. Bogaerts, D. Taillaert, L. O'Faolain, D. Van Thourhout and R. Baets, "Compact Focusing Grating Couplers for Silicon-on-Insulator Integrated Circuits", Photon. Technol. Lett., vol. 19, no. 23, pp. 1919-1921, 2007.

[4] S. Pathak, M. Vanslembrouck, P. Dumon, D. Van Thourhout and W. Bogaerts, "Optimized Silicon AWG with Flattened Spectral Response Using an MMI Aperture", J. Lightwave Technol., vol. 31, no. 1, pp. 87-93, 2013.

[5] T. Spuesens, S. Pathak, M. Vanslembrouck, P. Dumon, W. Bogaerts, "Integrated grating coupler/power splitter for on-chip optical power distribution", Group IV Photonics (GFP), 2014 IEEE 11th International Conference on, pp. 141-142, Aug 2014.

[6] D. Vermeulen, S. Selvaraja, P. Verheyen G. Lepage, W. Bogaerts, P. Absil, D. Van Thourhout and G. Roelkens, "high-efficiency fiber-to-chip grating couplers realized using an advanced CMOS-compatible siliconon-insulator platform" Opt. Express, vol. 18, no. 17, pp. 18278-18283, 2010 